\title{
A note on producing supercooled water in the laboratory
}

\author{
I. Amisaday Zarco-Delgado, and H. N. Núñez-Yépez \\ Departamento de Física, Universidad Autónoma Metropolitana, \\ Unidad Iztapalapa, Apartado Postal 55-534, Iztapalapa 09340 CDMX. \\ e-mail: zamisaday@gmail.com; nyhn@xanum.uam.mx \\ A. L. Salas-Brito \\ Laboratorio de Sistemas Dinámicos, Departamento de Ciencias Básicas, Universidad Autónoma Metropolitana, \\ Unidad Azcapotzalco, Apartado Postal 21-267, Coyoacán 04000 CDMX. \\ e-mail: asalasbrito@yahoo.com;asb@azc.uam.mx
}

Received 15 January 2021; accepted 9 March 2021

\begin{abstract}
As we lower the temperature of water at a certain point it changes from liquid to solid. Yet under certain conditions it is possible to keep the water in a non-equilibrium liquid-like phase called supercooled water thus avoiding solidification. In such a state water may freeze under the tiniest perturbation (as stirring it a little). The existence of supercooled water is usually known to students since the introductory courses, but it seems difficult for them to attest such a water state in an undergraduate laboratory. A simple method for supercooling water is, however, readily available. It is this short paper aim to describe such a method in a form that may be performed in any elementary laboratory.
\end{abstract}

Keywords: Supercooled water; undergraduate laboratories; metastable states.

PACS: 03.65.Pm; 03.65.Ge

DOI: https://doi.org/10.31349/RevMexFisE.18.020210

\section{Introduction}

Supercooled liquids, in particular water, have a long history dating back perhaps to the work of Joseph Black in the XVIII century [1].

Such liquids have a rich phenomenology which is still far from being completely understood. Water may enter the supercooled phase, that is, it still may be behaving as a liquid

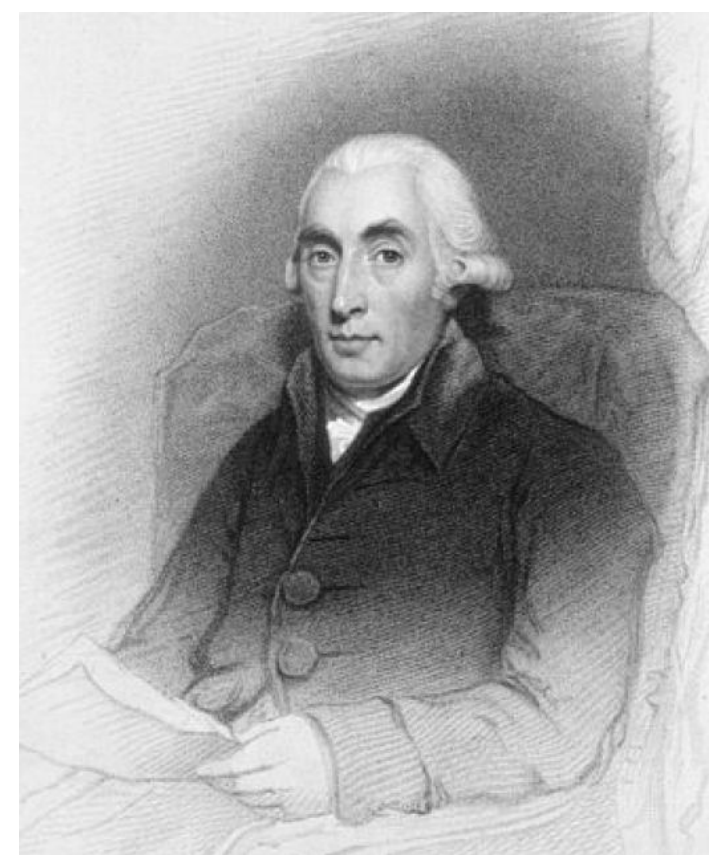

FIGURE 1. Joseph Black shortly before his death in 1799. well below its standard freezing point at $273.15 \mathrm{~K}$ (or $0^{\circ} \mathrm{C}$ ). Instead, it forms a curious substance, supercooled water, otherwise called undercooled or subcooled water. In fact, freezers can sometimes cool soft drinks to a supercooled state so that when they are opened form a kind of slush. Most people have witnessed this phenomena at home. Supercooling has many uses, for example it has been successfully applied to organ preservation at hospitals: livers that were later transplanted into recipient animals were preserved by supercooling for up to 96 hours (4 days), quadrupling the limits of what could be achieved by conventional methods.

For most substances, the melting and freezing points occur at the same temperature. However, certain substances possess differing solid-liquid transition temperatures. For example, agar (agar, also called agar-agar, a gelatinous substance obtained from various kinds of seaweed and used in biological culture media or in foods) displays hysteresis in its melting and freezing points: it melts at $85^{\circ} \mathrm{C}$ (or $358.15 \mathrm{~K}$ ) and solidifies at $32^{\circ} \mathrm{C}$ (or $305.15 \mathrm{~K}$ ). The properties of supercooled water may be also used to explain the winter dwellings of aquatic insects [2]. However, we do not address such matters in this work, requiring avanced explanations and a much more elaborate experiment for a basic undergraduate physics course. Though the idea of such a metastable water state is quite analogous to the equilibrium that may be reached by a broom-stick standing upright on the floor for one instant before falling down.

The crystallization of pure water usually begins at a lower temperature than the melting point, freezing does not start until the temperature is low enough to allow the formation of nucleation points, that is, small sites in which the freezing 
process may start. The melting point of water at one atmosphere of pressure is very close to $0^{\circ} \mathrm{C}$ (or $273.15 \mathrm{~K}$ ), and in the presence of nucleating substances, the freezing point of water is close to its melting point. In the absence of these, water may supercool to $-40^{\circ} \mathrm{C}(\simeq 233 \mathrm{~K})$ before freezing [3-7]. Anyhow, our aim here is not to discuss in any detail the properties of water at low temperatures, it is just to describe in the most direct way possible a method for obtaining supercooled water in an undergraduate laboratory [8]. Our method was found while trying to exhibit whether hot water may freeze quicker than cold, that is, in an activity intended to show the feasibility of the Mpemba effect [9-11]. What it is known is that supercooled water occurs in a variety of natural settings, including clouds, where liquid water has been observed at temperatures approaching $-40^{\circ} \mathrm{C}(233.15 \mathrm{~K})$ ! [5]. Water enters the supercooled phase when its temperature reaches below its freezing point at $0^{\circ} \mathrm{C}$ (or $\left.273.15 \mathrm{~K}\right)$ still behaving like a liquid. Other differences with standard liquid water is the manifest increment in the viscosity of the supercooled liquid which may be appreciated even visually as it adquires a jelly-like oily aspect-similar to the appearance of very cold vodka, see Fig. 3.

Once done with the preliminaries, the very simple procedure proposed for getting supercooled water in an elementary lab is detailed in the next section. You may see the process in the student taken video in https://www. youtube. com/watch?v=Xs1Ut 1mL OVw where some of the points mentioned above may be seen. The properties of supercooled water are hence of interest to both scientists and engineers. Supercooled water may be easily created in an elementary laboratory as we detail in what follows.

\section{How may we produce supercooled water?}

The process for obtaining supercooled water in the laboratory is particularly simple. For preparing the samples for the experiment, we collect $200 \mathrm{ml}$ of tap water in aluminum cans - of the kind used for baking. In one of the cans we add $200 \mathrm{ml}$ of room temperature $\left(\sim 20^{\circ} \mathrm{C}\right)$ tap water ${ }^{i}$; to the other, we add water at $74^{\circ} \mathrm{C}$. Take into account that the experiment was performed in Mexico City where water boils at approximately $96^{\circ} \mathrm{C}$ as a result of the city's altitude of roughly $2240 \mathrm{~m}$ ). The cans were next put inside two cilindrical metallic trays filled with crushed ice heavily sprinkled with salt which reached a minimum temperature of $-12^{\circ} \mathrm{C}$ -as measured with a mercury laboratory thermometer. The temperature of the cans was measured every 2 minutes using two of these thermometers left inside cans. See Fig. 2 for the experimental set up, Fig. 3 for a photograph of a sample of supercooled water, and Fig. 4 for $T$ vs time plots of the experimental results. The choosing of tap water introduces an interesting query, as it is known that the supercooling process depends strongly on the purity of the water sample. We have not observed any effect of this kind $[3,4]$ in the several experiments the students have conducted -but, everything we

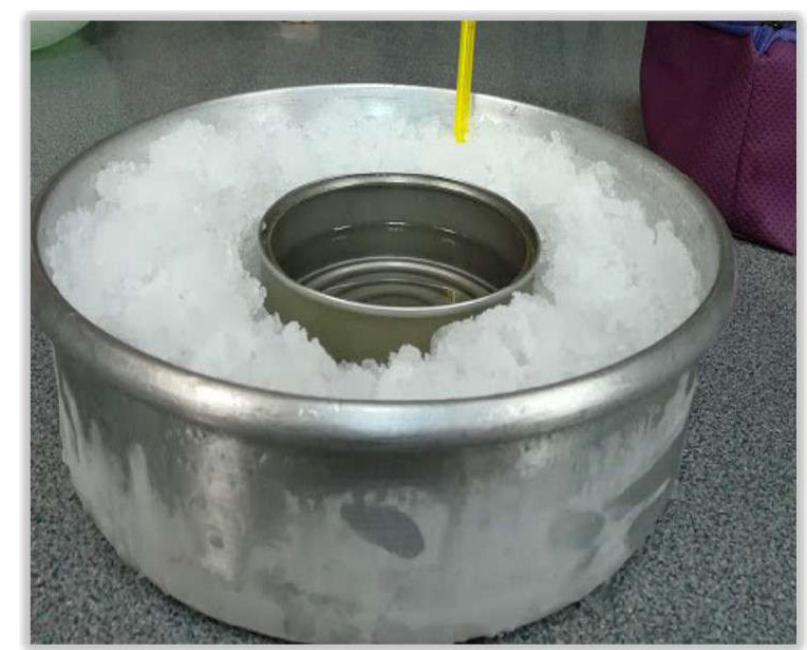

FIGURE 2. The experimental arrangement: cans with water surrounded by salted crushed ice and a mercury laboratory thermometer for measuring the temperature of the ice.

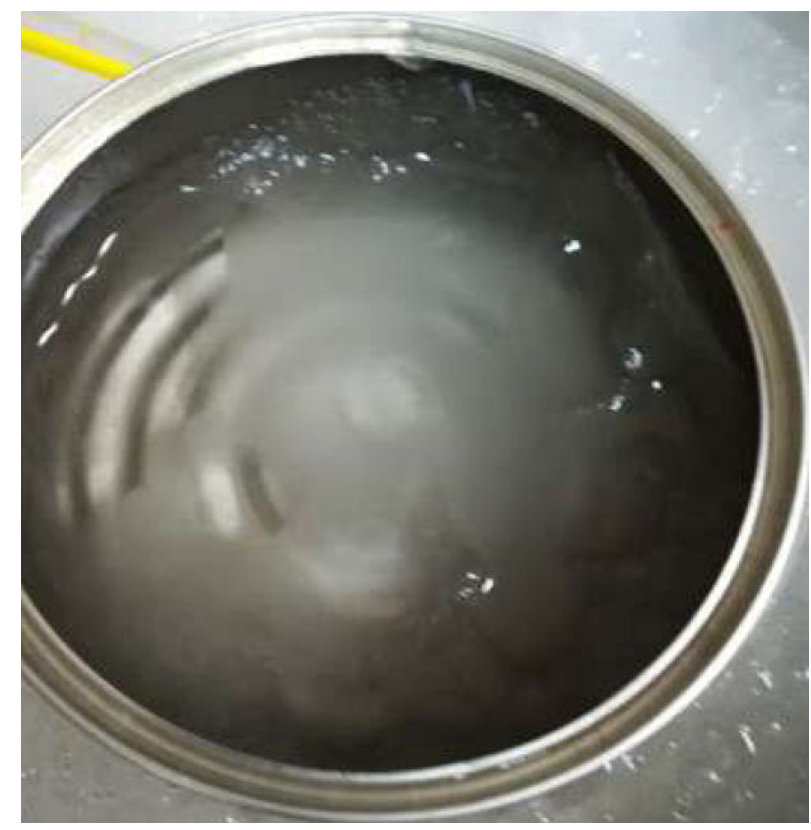

FIGURE 3. Supercooled water.

say here was performed by undergraduates working in a teaching lab- we have not an explanation of this fact, unless we believe in the great purity of the University's tap water.

With this simple arrangement we observe the almost instantaneous freeezing of the water surface on reaching $T=$ $-4.2^{\circ} \mathrm{C}$, while the rest of the water adquired a sort of jellylike texture before freezing. In fact, in these conditions the supercooled liquid became solid upon any perturbation, for example, as a result of moving the can or perturbing it with the thermometer. You may see this effect in the student taken video referred to above or in Fig. 3.

We should mention the sudden rise in temperature that occurs on freezing, the temperature of the sample rises from 


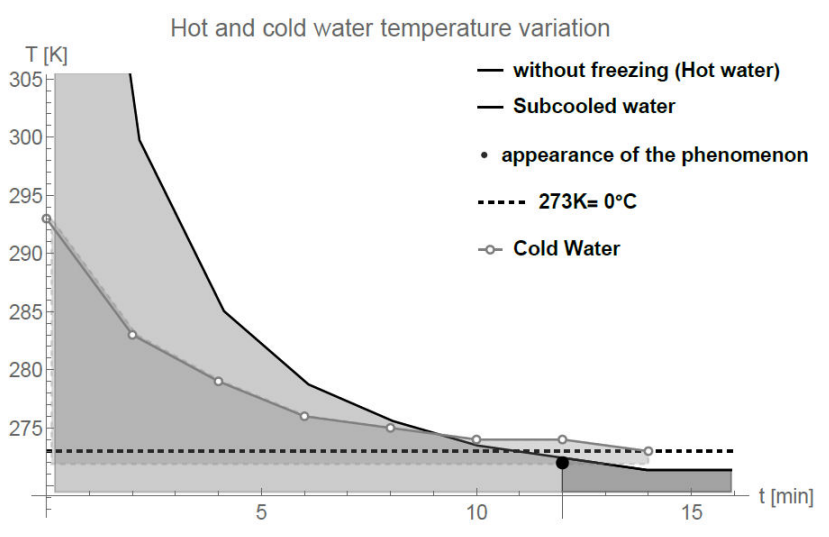

FIGURE 4. Temperature variation with time, temperature is given in kelvin while time is in minutes. The point marked with a black dot shows the state where the photograph (Fig. 3) of the supercooled state was taken. Incidentally, the intersection of the hot and cold water lines before freezing exhibits that in this experiment the Mpemba effect does indeed occur.

$T_{i}=-4.2^{\circ} \mathrm{C}$ to $T_{f}=0^{\circ} \mathrm{C}[3,4]$. This sudden rise is not shown in any of the plots displayed in this paper.

The time it takes to lower the water temperature, that is, the time interval in which the water reached its freezing point was from 5 to 6 minutes.

Giving the surprising outcome of our proposed activity for cooling water, we remark again that water has indeed remarkable physical properties that differ from most other common liquids. In the ambient temperature regime, in which we may classify our proposed method, its properties already deviate from those of any other simple liquid. We may assert that water is one of the most interesting materials in the universe both in its solid as well as in its liquid phase. Water is a material that we see a lot and hence it mostly does not cross our mind that water is rather special. Let us mention here a few of its properties, markedly differing from comparable materials, a) water has a high melting point, b) it has a high boiling point, c) high surface tension, d) high viscosity (that moreover decreases with increasing pressure), e) during melting it increases its specific density, f) between $0{ }^{\circ} \mathrm{C}$ and $4{ }^{\circ} \mathrm{C}$ its density increases with temperature, and g) it can be supercooled in the form of small drops until it reaches $-70^{\circ} \mathrm{C}[4,6]$.

\section{Conclusion}

We have described in this work a very simple method for obtaining supercooled water in an undergraduate laboratory and mentioned some of the differences of this metastable state from standard ice and from similar properties of other substances. We also expect it to be clear by now that water or any other liquid is said to be supercooled when it remains at the liquid state at and below its melting temperature. A supercooled state is a non-equilibrium state with a finite lifetime where the system is trapped in a relative minimum of the appropriate (thermodynamic) potential. The greatest in- terest in studying supercooled water is perhaps the light that it can shed on the nature of liquid water and we cannot forget the various applications of the phenomenom from the purely amusing as in the spontaneous making of slurs in iced soft drinks, to the serious medical ones as in preserving organs for transplanting. Such uses makes supercooling important for both technology and physics teaching.

Keeping water in its supercooled regime may depend on various factors such as the purity of the sample and the absence of external mechanical or thermal perturbations. However, at certain thermodynamic conditions water cannot be kept supercooled anymore and two possible outcomes of the supercooled phase may occur: a) ice crystallization or b) a glass transition. In the first case, a first order phase transition from liquid to solid takes place and nucleation drives the liquid in the stable ice phase. In the second, through a rapid quenching of the liquid to a sufficiently low temperature ice nucleation is prevented. The supercooled liquid reaches a state where the molecules are structurally arrested: the liquid becomes a glass. Giving the elementary nature of the lab, we never attempted to verify in any way the properties of the supercooled water obtained.

The supercooling of water also gives rise to a conundrum since a rain drop in winter freezes from the outside in and hence it presents a difficulty: the expansion of water upon freezing is incompatible with self-confinement by a rigid ice shell. This problem was recently solved in [12] where it is argued that the phenomena may be prevented by the effect of surface tension in submillimetric (sizes below $50 \mu \mathrm{m}$ ) water droplets which prevents the disruptive expansion. Another problem, noticed by us, is the apparent lack of dependence of the supercooling process with the purity of the water sample, at least if we take into account that the tap water at the University undergraduate laboratories may not be the purest and that the substances diluted in it may change a little on a daily or a weekly basis.

The experimental results obtained may be regarded as part of what makes water so important for the existence of life on Earth and for the amusement of scientists. Whatever the case may be, we think this method for obtaining supercooled water was quite interesting for all the students attesting it. One of the objectives of this work is to exhibit the easiness in which we can perform experimental activities with rather unexpected results even in undergraduate labs. Such unexpected outcomes may contribute to motivate scientists or engineers in the making to select the experimental approach for the study of nature.

\section{Acknowledgements}

Thereferences provided by our colleague C. A. Vargas are gratefully acknowledged. The portrait of J. Black was taken from the Wikipedia for which it must receive our warmest thanks. Thanks must be also extended to M. S. Chato, C. H. Ray, Z. Gris, F. D. Samy, M. S. Kuroi, G. O. Pelucidar, and G. V. Mitzu for their helpful comments on the manuscript. 
$i$. Starting here, we stop using both ${ }^{\circ} \mathrm{C}$ and $\mathrm{K}$ to give temperatures.

1. J. Black, The supposed effect of boiling upon water, in disposing it to freeze more readily, ascertained by experiments, Phil. Trans. R. Soc. 65 (1775) 124, https : // doi.org/10. $1098 /$ rstl.1775.0014

2. R. W. Bouchard, B. E. Schuetz, L. C. Ferrington, and S. A. Kells, Cold hardiness in the adults of two winter stonefly species: Allocapnia granullata (Claassen, 1924) and A. pygmaea (Burmeister, 1839) (Plecoptera: Capniidae), Aquat. Insects 31 (200) 145, https : / / doi . org/10. $1080 / 01650420902776690$.

3. A. Cavagna, Supercooled liquids for pedestrians, Phys. Rep. 476 (2009) 51, https://doi.org/10.1016/j. physrep.2009.03.003

4. K. Hwan Kim et al., Maxima in the thermodynamic response and correlation functions of deeply supercooled water, Science 358 (2017) 1589, https://doi.org/10.1126/ science.aap8269

5. K. Sassen, K. Nan Liou, S. Kinne, and M. Griffin, Highly Supercooled Cirrus Cloud Water: Confirmation and Climatic Implications, Science 227 (1985) 411, https : / / doi . org/ $10.1126 /$ science.227.4685.411
6. P. Gallo and H. Eugene Stanley, Supercooled water reveals its secrets, Science 358 (2017) 1543, https : / doi.org/10. $1126 /$ science.aar3575

7. P. Gallo et al., Water: A Tale of Two Liquids, Chem. Rev. 116 (2016) 7463, https://doi.org/10.1021/acs. chemrev.5b00750

8. I. W. Geer, Weather Talk for Teachers and Students of the Weather: A Simple Technique For Supercooling Water, Weatherwise 32 (2010) 84, https : / / doi.org/10 .1080/ 00431672.1979 .9931872 .

9. L. Kurić, Why Hot Water Freezes Faster than Cold?, Int. Lett. Chem. Phys. Astron. 32 (2014) 119, https://doi.org/ $10.18052 /$ www.scipress.com/ILCPA.32.119

10. E. B. Mpemba and D. G. Osborne, Cool?, Phys. Educ. 4 (1969) 172, https://doi.org/10.1088/0031-9120/4/3/ 312

11. D. G. Osborne, Mind on ice, Phys. Educ. 14 (1979) 414, https://doi.org/10.1088/0031-9120/14/7/ 313

12. S. Wildeman, S. Sterl, C. Sun, and D. Lohse, Fast Dynamics of Water Droplets Freezing from the Outside In, Phys. Rev. Lett. 118 (2017) 084101, https://doi.org/10.1103/ PhysRevLett.118.084101 